\title{
El Rol Económico de los Recursos Malacológicos durante el Holoceno Medio en la Costa Arreica del Norte de Chile: Sitio Agua Dulce
}

\author{
The Economic Role of Malacological Resources during the Middle Holocene in the \\ Interfluvic Coast of Northern Chile: The Site Agua Dulce
}

Laura Olguín ${ }^{\mathrm{i}}$

\begin{abstract}
RESUMEN
Los restos malacológicos más abundantes en el registro arqueológico del sitio Agua Dulce $\left(25^{\circ}\right.$ Lat. S) son los gastrópodos, especialmente Concholepasconcholepas y Fisurella spp. Estas especies han sido recolectadas profusamente desde el 9.000 cal. años AP, y en especial a partir del Holoceno medio (7.000 - 5.000 años cal.AP) cuando el registro arqueológico local muestra un sistema de asentamiento de baja movilidad residencial. Los tamaños pequeños y medianos de las conchas presentes en el registro evidencian una estrategia de recolección de fácil acceso y no selectiva de individuos juveniles aún no reproductivos, ubicados a lo largo del intermareal rocoso.
\end{abstract}

Palabras claves: Recursos malacológicos, Tamaño, Holoceno Medio, Economía Costera.

\section{ABSTRACT}

The most abundant malacological remains in the archaeological record of the site Agua Dulce ( $25^{\circ}$ Lat. S) malacological remains are gastropods, especially Concholepasconcholepas and Fisurellas spp. These species have been widely collected since $9000 \mathrm{cal} . \mathrm{yr} \mathrm{BP}$, and especially from the mid-Holocene $(7,000-5,000$ years cal BP) when the local archaeological record shows a system of settlement of low residential mobility. Small and medium sizes of shells present in the record show an accessible and non- selective collection strategy of nonreproductive juveniles located along the rocky intertidal.

Key Words: Resources malacological, Size, Mid-Holocene, Coastal Economy.

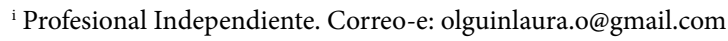

Recibido: 29-12-2012 - Revisado: 09-082013 - Aceptado: 08-01-2014 


\section{INTRODUCCIÓN}

Dentro de las diferentes clases de depósitos con que se trabaja en arqueología, los conchales ofrecen una serie de posibilidades hasta ahora poco aprovechadas para la reconstrucción del modo de vida de las sociedades costeras, sobrestimando frecuentemente su volumen $y$ generando interpretaciones inapropiadas sobre la dieta y tiempo de permanencia de las personas en los asentamientos (Bailey 1975, Perlman 1980,Yesner 1980, Waselkov 1987, Erlandson I 988 y 200I).

Es así como el rol económico de los moluscos en una sociedad costera puede tener grandes implicancias para la interpretación sobre la estructura de una población, el sistema de subsistencia o la evolución de las adaptaciones costeras en general (Erlandson 1988). De esta manera, un estudio sistemático de este tipo de depósitos, compuestos por innumerables restos como conchas y óseos descartados, provee una valiosa fuente de información para replantearnos las aseveraciones y generalizaciones propuestas.

Modelos de forrajeo óptimo, de capacidad de carga de especies biológicas y estudios sobre explotación humana de recursos costeros (Braje y Erlandson 2009, Hockey y Bosman 1986, Oliva y Castilla 1986) predicen que la recolección constante y sostenida de individuos, previo a su edad reproductiva, genera una progresiva disminución de la productividad de éstos, y por lo tanto, una reducción en las abundancias y tamaños de los recursos malacológicos disponibles. La disminución en el tamaño de las conchas, entonces, ha sido una de las herramientas más usada en arqueología para interpretar impacto de la recolección humana en algunas especies de moluscos (Brajeet al. 2007, Erlandsonet al. 2008, Rick y Erlandson 2008).

A partir de lo anterior, consideramos que para comprender el rol de los moluscos y potencialidad como recurso económico resulta fundamental determinar las tendencias de tamaños de los mismos, así como las características biológicas, su distribución y hábitat de las especies recolectadas (Waselkov 1987). Sólo de esta forma, podremos reconocer ciertas variaciones dentro de las estrategias de explotación de moluscos, ya sea por impacto de cambios medioambientales o excesiva recolección humana.

A pesar de los diversos usos tecnológicos sugeridos para la porción no comestible de los moluscos (la concha), el estudio malacológico que se presenta a continuación, se enfoca substancialmente en analizar la variación cronológica en el tamaño y abundancia de conchas de Concholepas concholepas y Fisurellas sp.con el fin de evaluar su rol y potencialidad en la economía costera del sitio Agua Dulce, y sus posibles evidencias de impacto humano en los ecosistemas explotados durante el Holoceno medio en la costa del Desierto de Atacama.

\section{EL CONTEXTO ARQUEOLÓGICO}

El sitio Agua Dulce se ubica en los $25^{\circ}$ Lat. $\mathrm{S}$ del litoral arreico e hiperárido de Chile (Figura I) y corresponde a un extenso conchal $\left(70.000 \mathrm{~m}^{2}\right)$ emplazado en un antigua terraza de erosión marina (6 msnm), con fácil acceso al borde litoral actual. El asentamiento es definido como un campamento residencial con una ocupación semipermanente, orientado básicamente al aprovechamiento humano de recursos marinos (Olguín 20I I).

El contexto arqueológico del sitio muestra un depósito estratigráfico con una potencia de $130 \mathrm{~cm}$ que representa una secuencia ocupacional de grupos cazadores, recolectores y pescadores del Holoceno medio, y una posterior reocupación efímera por grupos alfareros y posthispanos. Los depósitos estratigráficos correspondientes al Holoceno medio contemplan al menos cuatro eventos ocupacionales fechados entre los 6.258 a 6826 cal. AP' (Figura I), los cuales están constituidos por la acumulación de restos de fauna marina, compuesta básicamente por otáridos, delfínidos, cetáceos, moluscos y peces, mientras que la fauna terrestre incluye camélidos y aves, aunque en menor frecuencia. El instrumental para la captura y procesamiento de estos recursos incluye puntas de proyectil, barbas de arpón, pesas y anzuelos, raederas, cuchillos, entre otros (Olguín 20II). 


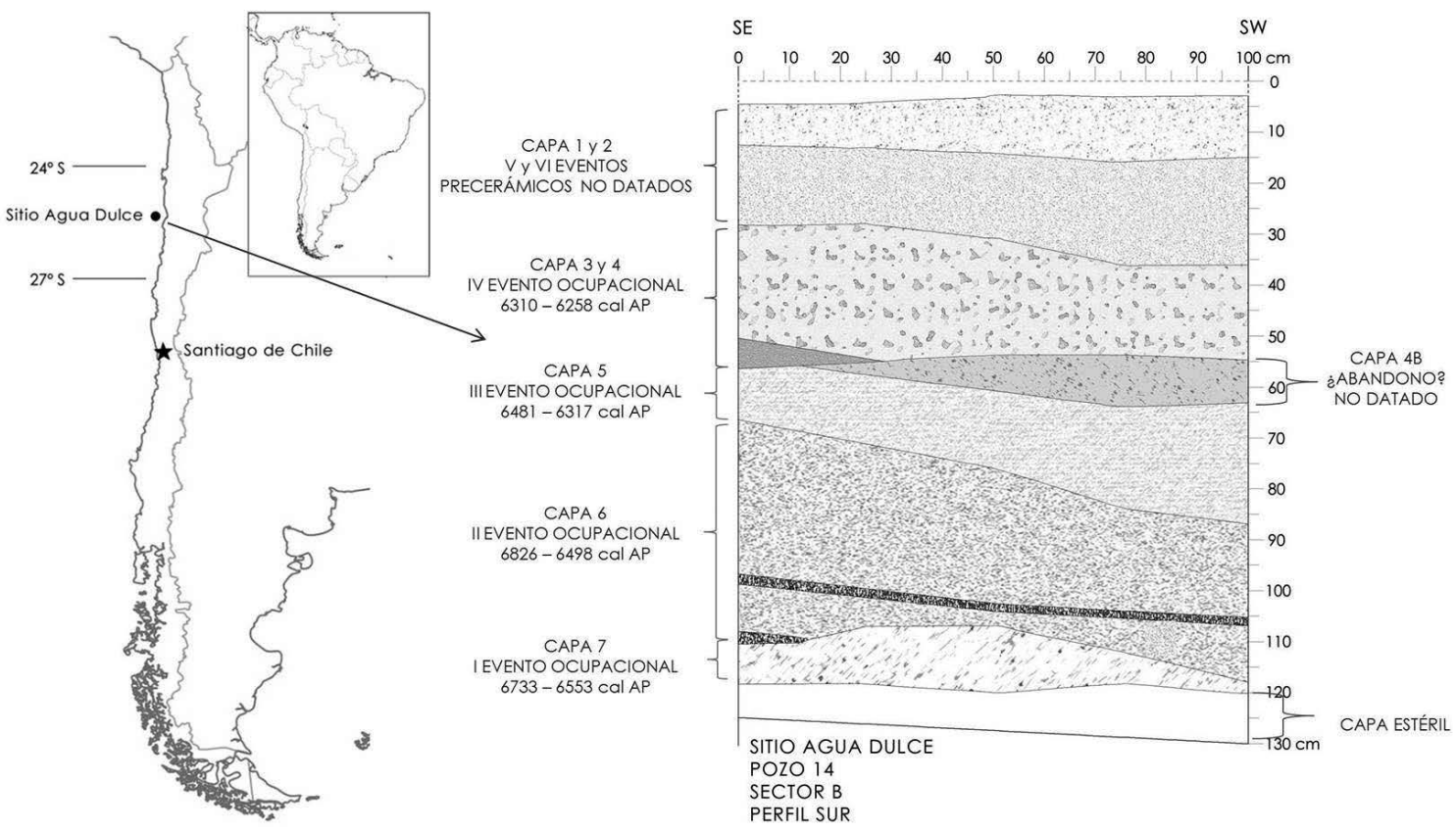

Figura 1: Localización área de estudio y perfil estratigráfico del sitio Agua Dulce.

Figure 1: Location of study area and stratigraphic profile from the site Agua Dulce.

\section{MATERIALESY MÉTODOS}

Los recursos malacológicos provienen de una columna de control de fauna de 50 $x 50 \mathrm{~cm}$ y $130 \mathrm{~cm}$ de profundidad, la cual fue harneada en laboratorio con un tamiz de I $\mathrm{mm}$, con la finalidad de obtener una muestra completa y representativa de los restos dentro de una dimensión controlada (Zohar y Belmaker 2005). El análisis consideró no sólo las conchas de moluscos sino que también los restos de crustáceos y equinodermos, constituyendo una muestra con abundante presencia de valvas enteras y restos fragmentados.
Para estimar la abundancia taxonómica y anatómica en el conjunto malacológico se utilizó el NIPS (Número de Especímenes Identificados) y el MNI (Número Mínimo de Individuos) (Grayson 1984,Lyman 1994). El NIPS incluye todos los restos malacológicos identificables anatómicamente para cada taxón. El MNI corresponde a la estimación del número de individuos para cada taxón y fue calculado tomando la unidad anatómica más representada como umbo, orificio apical y columela en gastrópodos; primera u octava placa en gastrópodospoliplacóforos; lateralidad de las charnelas en bivalvos; los dactilopoditos en crustáceos; y la linterna de Aristóteles en equinodermos (Claassen 1998, Jerardinoet al. 1992) (Figura 2). 


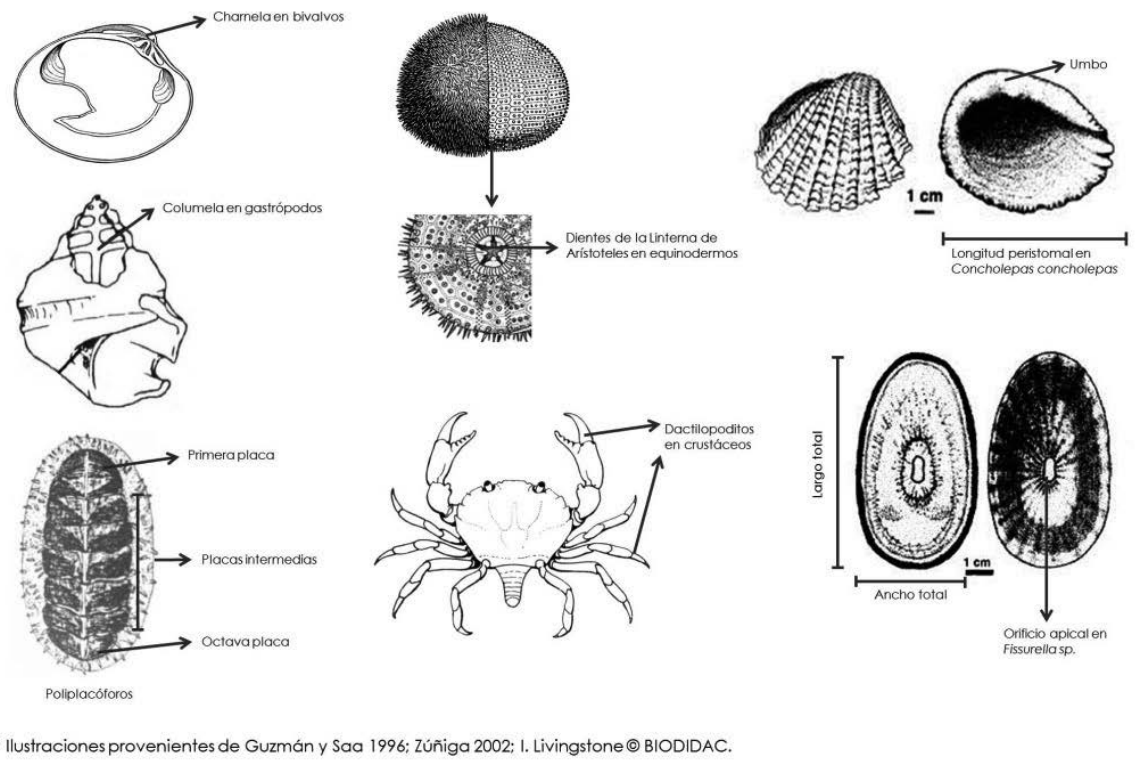

Figura 2: Unidades anatómicas diagnósticas para la estimación de las medidas morfométricas y cálculo de MNI.

Figure 2: Diagnostic anatomical units used to estimate the morphometric measurements and calculation of MNI.

Además, utilizando un caliper vernier se estimó la talla sobre la base del largo en aquellas especies de moluscos con mayor importancia económica: Concholepas concholepas, Fissurella limbata y Fissurella crassa (Figura 2). En caso de ausencia del largo para Fisurélidos, se aplicó un análisis de regresión, elaborado a partir de una ecuación proveniente de especímenes completos del mismo conjunto arqueológico (Oliva y Castilla 1992). Una vez obtenidas todas las medidas morfométricas se obtuvo la Media Estadística a través del Programa SPSS Statistics 20, con el fin de comparar el tamaño de los distintos taxa a lo largo de la secuencia ocupacional del sitio.

\section{RESULTADOS}

Diversidad taxonómica, frecuencia y distribución del conjunto malacológico

De un total de 10.069 especímenes (NISP), se calculó un $\mathrm{MNI}$ de 5.129 y una diversidad taxonómica de $5 \mathrm{I}$ especies. A juzgar por su gran biomasa cárnica, abundancia en concentración, predictibilidad y fácil acceso, los invertebrados marinos más representados corresponden a las familias de gastrópodos Trochidae, Fissurelidae, Muricidae y Chitonidae correspondientes al Phylum Mollusca (Figura 3). Los ejemplares de la familia Trochidae (Tegulasp.) representan la mayor abundancia, con una altísima concentración en el IV evento ocupacional adscrito entre los 6.310 a 6258 cal. AP. La frecuencia de individuos pertenecientes a las familias Muricidae (Concholepas concholepas), Fissurelidae (Fissurella sp.) y Chitonidae (Acanthopleurae chinata) se distribuye homogéneamente durante todos los eventos ocupacionales del Holoceno medio, no revelando variaciones significativas a excepción de la frecuencia de Tegula sp. No obstante, se observa una disminución significativa en la frecuencia de estas especies tanto en la ocupación más temprana del sitio (Evento I) como en la más tardía (Evento Vl). 


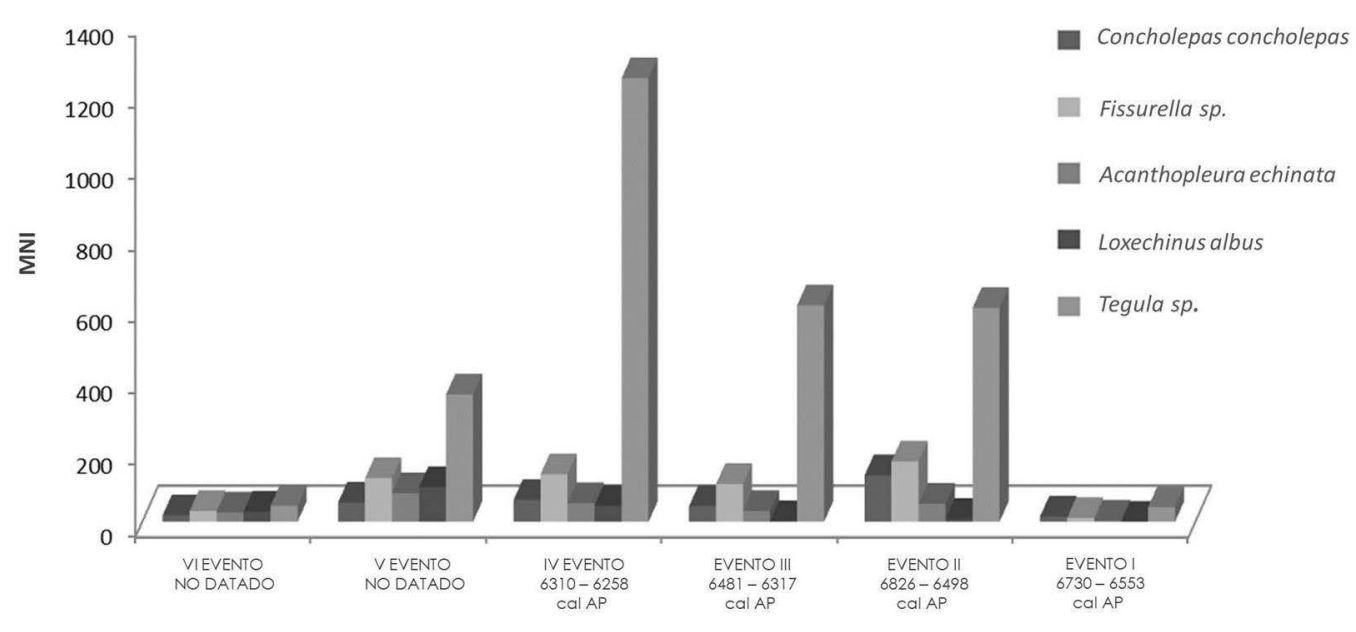

EVENTOS OCUPACIONALES

Figura 3: Frecuencia de recursos malacológicos con mayor importancia económica a lo largo de la secuencia ocupacional del sitio Agua Dulce.

Figure 3: Frequency of most economically important malacological resources along occupational sequence at site Agua Dulce.

Otras variaciones en el comportamiento distribucional de las especies a lo largo de la secuencia se relaciona con la abundancia de equinodermos, pues éstos se concentran altamente en el $V$ evento ocupacional, disminuyendo significativamente hacia las ocupaciones más tempranas del Holoceno medio (Figura 3). Caso contrario ocurre con los bivalvos Prototha cathaca y Choromytilus chorus, que se concentran exigua, pero preferentemente en los eventos II y III.

La frecuencia de crustáceos (Phylum Crustacea) también resultó baja en todos los eventos del sitio, no superando los II especímenes, anatómicamente representados sólo por algunos dactilopoditos.Probablemente esta escasa frecuencia se deba a problemas de conservación diferencial, dado que el material orgánico que contempla gran parte del exoesqueleto de los decápodos tiende a ser muy frágil, preservándose aquellas partes anatómicas más resistentes y con mayor contenido calcáreo.

En suma, los gastrópodos representaron el $95 \%$ del $\mathrm{MNI}$ total registrado. Probablemente la mayoría de ellos fueron seleccionados para el consumo, destacándose las especies Tegula atra, Fissurella crassa, Fissurella limbata, Concholepas concholepas y Acanthopleura echinata. El porcentaje restante corresponde a caracoles pequeños de captura no intencional como Turritela cingulata, Scurria parasitica, Diloma nigérrima, Nasarius gayi, entre otros, integrándose al contexto arqueológico por procesos naturales, como por ejemplo el arrastre al extraer otros recursos marinos.

Rangos de tamaño de las especies de mayor importancia económica

Se tomaron las medidas morfométricas de 319 ejemplares, de los cuales $50.1 \%$ corresponde a Concholepas concholepas, el 19,7\% a Fissurella crassa y el $30.1 \%$ a Fissurella limbata. Para Concholepas concholepas se obtuvo un tamaño con una media de $52 \mathrm{~mm}$. (Figura 4a), considerándose relativamente pequeño, pues el tamaño máximo de un individuo adulto puede alcanzar una longitud de $150 \mathrm{~mm}$ (Osorio 2002). Estos resultados muestran que durante el Holoceno medio existiría una tendencia de captura enfocada preferentemente en individuos juveniles no reproductivos y recolectados fácilmente a la baja marea, adheridos a la parte baja de las rocas (Guzmán y Saa, 1996). Esta talla se mantuvo constante a lo largo del Holoceno medio, pues no observan grandes variaciones morfométricas. 

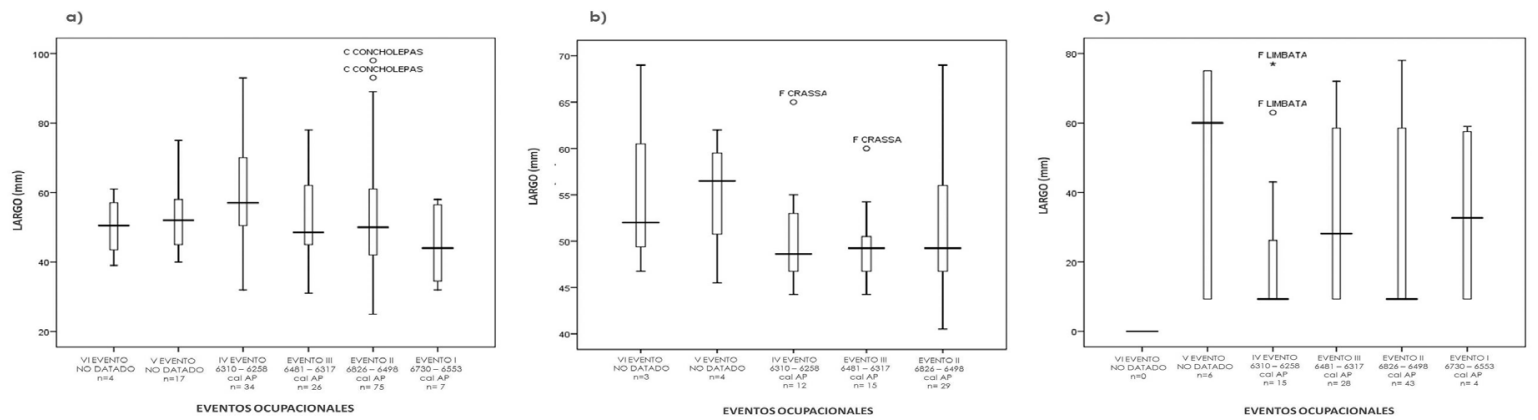

Figura 4:Variación del tamaño en: a) Concholepasconcholepas; b) Fissurella crassa;y c) Fissurella limbata a lo largo de la secuencia ocupacional del sitio Agua Dulce.

Figure 4: Size Variation: a) Concholepasconcholepas; b) Fissurella crassa; and c) Fissurella limbata along occupational sequence at site Agua Dulce.

El tamaño medio en Fisurélidos también reveló un comportamiento similar (Figura $4 \mathrm{~b}$ y c), pues las dos especies consideradas no alcanzaron su tamaño adulto al momento de su captura, obteniéndose una media de $49.24 \mathrm{~mm}$ para Fissurella crassa,y $56.96 \mathrm{~mm}$ para Fissurella limbata. El rango de talla adulta de ambas especies fluctúa entre los 60 y 90 mm (McLean 1984), lo que sugiere una extracción fácil desde las rocas expuestas al oleaje a nivel de la baja marea para Fissurella crassa (Osorio et al. 1979 en Guzmán y Saa 1996, Oliva y Castilla 1986), y bajo el cordón de Perumytilus purpuratus (chorito maico) hasta el nivel inferior de las algas pardas para Fissurella limbata (Oliva y Castilla 1992, Zúñiga 2002).

\section{DISCUSIÓN}

Del conjunto malacológico analizado a pesar que se observa una alta variabilidad de recursos que habitan principalmente en el intermareal rocoso, que es sin duda la zona más rica y productiva del sistema costero, es posible identificar que el principal volumen de la muestra consiste solamente en cinco especies buscadas como recurso alimenticio: Concholepas concholepas ("loco"), Fissurella sp. ("lapa"), Tegula atra ("caracol negro"), Acanthopleura echinata ("chitón o apretador") y Loxechinus albus (erizo).A juzgar por su alto rendimiento cárnico y biomasa, dichas especies representan a los moluscos de mayor importancia económica para los ocupantes del sitio Agua Dulce durante el Holoceno medio.

Sobre la base del historial de vida de los gastropódos analizados, las tendencias de talla mediana a pequeña registradas para Fisurella spp. y $C$. concholepas indicarían que el grado de accesibilidad para la recolección de los recursos malacológicos por parte de los ocupantes del sitio estaría delimitado desde el intermareal hasta el submareal somero (6-12 metros de profundidad para tallas máximas), pues existiría una clara variación de la ubicación en profundidad del molusco a medida que avanza su ciclo de vida (Báez et al. 2004, Guzman y Saa 1996, Osorio 2002).

En este sentido, la media de tamaño obtenida revela que su extracción se habría realizado al finalizar su etapa juvenil, pudiendo ser efectuada fácilmente desde la orilla o en momentos de la baja marea, dando cuenta de una explotación de recursos no especializada mediante el uso de tecnologías simples que puede ser efectuada por todos los grupos etáreos (Yesner 1980) y no requiriendo un gasto energético muy elevado (Perlman 1980).

Ahora bien, las inferencias esperables para el conjunto analizado serían que la media de los tamaños disminuyera debido a la recolección constante y sostenida de individuos previo a su edad reproductiva, o bien, esta media aumentará, ya que al disminuir la abundancia de individuos más accesibles, se explorarían nuevos hábitat como la 
zona submareal, donde hay ejemplares de mayor tamaño. Sin embargo, los resultados registran que las medias de tamaño son más bien constantes a través del tiempo, pues no se evidencia un patrón de disminución ni de aumento de tamaños. En este sentido, no existiría un uso diferencial de este recurso ni tampoco un impacto humano en el ecosistema explotado durante los eventos ocupacionales del Holoceno medio, reflejando una recolección constante y sostenida de gastrópodos durante los dos mil años de ocupación del sitio de estudio.

No obstante, consideramos necesario que determinar la intensidad de ocupación de cada evento nos podrá proporcionar mayor información sobre las fluctuaciones de tamaño en los moluscos, pues de este modo podremos evaluar cómo la presión humana altera las tasas de crecimiento de estas especies.

Sin duda alguna, entonces, el rol económico de los recursos malacológicos en el sitio de estudio se fundamenta en ser el alimento básico de la dieta $y$ en un potencial elemento de estabilidad en una economía cazadora-recolectora. No obstante, esto no implica una dependencia básica en los moluscos como alimento, sino que éstos actuarían como un complemento alimenticio de otros recursos como otáridos y peces (Tabla I).

\begin{tabular}{|l|l|l|}
\hline RECURSOS FAUNÍSTICOS & MNI & NIPS \\
\hline Moluscos - Crustáceos - Equinodermos & 5129 & 10069 \\
\hline Peces & 673 & 12860 \\
\hline Mamíferos marinos & 12 & 136 \\
\hline Mamíferos terrestres & 6 & 69 \\
\hline Aves marinas & 10 & 38 \\
\hline
\end{tabular}

Tabla 1: Abundancia relativa de los recursos nutricionales costeros provenientes de la columna de fauna.

Table 1: Relative abundance of nutritional resources from the coastal control column.

Por tanto, a pesar de que el volumen de valvas de moluscos en los conchales tenga una tasa de desperdicio mayor (densa cantidad de conchas en los depósitos) en relación a la porción no productiva de otros recursos más nutritivos (óseos de peces o mamíferos), su porcentaje nutricional calórico y cárnico siempre será bajo (Bailey 1975, Orquera 1999). Una dieta fundada únicamente sobre moluscos es pobre en calorías para los requerimientos metabólicos de un ser humano (Yesner 1980), y no podría sustentar a largo plazo más que a unos pocos individuos (Bailey 1975).

\section{AGRADECIMIENTOS:}

Este trabajo se enmarcó dentro del Proyecto FONDECYT 1080666, agradeciendo a todo el personal humano amante de la costa, en especial a Pedro Andrade y Carola Flores.

Notas

' Los fechados I4C del sitio Agua Dulce fueron calibrados con dos sigmas utilizando la curva INTCAL09 del Programa CalibRadiocarbonCalibration Versión 6.0, expresándose todas las edades en años calendario antes del presente (A.P.).

\section{BIBLIOGRAFÍA}

Báez, P., J- Arata y D. Jackson. 2004. "El Loco Concholepasconcholepas (Bruguiere, 1789) (Mollusca: Gastropoda: Muricidae) como recurso durante el Holoceno Temprano-Medio en Los Vilos, Chile Central". Investigaciones Marinas 32 (I): I07II3.

Bailey, G. N. 1975. "The role of molluscs in Coastal Economies: The results of midden analysis in Australia". Journal of Archaeological Science 2: $45-62$.

Braje, T.J., D.J. Kennett, J. M. Erlandson y B. J. Culleton.2007. "Human impacto on nearshore shellfish taxa: a 7,000 year record from Santa Rosa Island, California". American Antiquity 72 (4): 735 - 756.

Braje, T. J. y J. M. Erlandson. 2009. "Molluscs and Mass Harvesting in the Middle Holocene: Prey Size and Resource Ranking on San Miguel Island,Alta California”. California Archaeology I (2): 269 - 289.

Claassen, C.1998. Shells. Cambridge Manuals in Archaeology. Cambridge University Press, Cambridge.

Erlandson, J. M. I988. "The role of shellfish in prehistoric economies: A protein perspective”. American Antiquity 53: 102 109.

--- 200I. "The archaeology of aquatic adaptations: Paradigms for a new millennium". Journal of Archaeological Research 9: 287-350.

Erlandson, J. M., T. C. Rick, T. J. Braje, A. Steinberg y R. L. Vellanoweth. 2008."Human impacts on ancient shellfish: A 10,000 year record from San Miguel Island, California”. Journal of Archaeological Science 35: 2144 - 2152.

Grayson, D. 1984. Quantitative Zooarchaeology. Academic Press, Orlando.

Guzmán, N y S. Saa. 1996. Taxonomía, distribución y principales características ecológicas de la malacofauna de la Región de Antofagasta. Seminario para optar al Grado Académico de Licenciado en Ciencias del Mar y al Título Profesional de Ingeniero en Acuicultura. Universidad de Antofagasta. Antofagasta. 
Hockey P. y A.L. Bosman.1986. "Man as an intertidal predator in Transkei: disturbance, convergence and management of a natural food resource". Oikos 46: 3 - I4

Jerardino, A., J.C. Castilla, J.M. Ramírez yN. Hermosilla. 1992.“Early coastal subsistence patternsin Central Chile. A systematic study of the marineinvertebraty fauna from the site of Curaumilla I". Latin American Antiquity 3(I): 43-62.

Lyman, R. L. 1994. Vertebrate Taphonomy. Cambridge Manuals in Archaeology. University Press, Cambridge.

Mc Lean, J. 1984. "Systematics of Fissurella in the Peruvian and Magellanic Faunal Provinces (Gastropoda: Prosobranchia)". Contributions in Science 354.

Olguín, L. 201 I. Historia de un conchal: Procesos de Formación y Secuencia Ocupacional del Sitio Arqueológico Agua Dulce, Costa Arreica del Desierto de Atacama, Comuna de Taltal, Región de Antofagasta. Tesis de grado para optar al Título de Arqueólogo. Facultad de Ciencias Sociales, Universidad de Chile. Santiago, Chile.

Oliva, D. y J.C. Castilla. 1992. "Guía para el Reconocimiento y Morfometría de Diez Especies del Género Fisurellas Bruguiere 1788 (Mollusca: Gastropoda) Comunes en la Pesquería y Conchales Indígenas de Chile Central y Sur". Gayana Zoológica 56 (3-4): 77-108.

---1986."The effect of human exlusion on the population structure of the keyhole limpets Fissurella crassa and F. limbata on the coast of Central Chile". Marine Ecology 7:20I - 217.
Orquera, L.A. 1999. "El consumo de moluscos por lo canoeros del extremo sur". Relaciones de la Sociedad Argentina de Antropología XXIV: 307 - 327.

Osorio, C. 2002. Moluscos marinos en Chile: especies de importancia económica. Guía para su identificación. Facultad de Ciencias. Universidad de Chile.

PerIman, S.M. 1980. "An optimum diet model, coastal variability and hunter-gatherer behavior". Advances in Archaelogical Method and Theory 3: 257-310.

Rick, T. C. y J. M. Erlandson. 2008. "Archaeology, historical ecology, and the future of ocean ecosystems". En Human Impacts on Ancient Marine Ecosystems: A Global Perspective, editado por T. C. Rick y J. M. Erlandson, pp: 297-307. University of California Press, Berkeley.

Waselkov, G. 1987."Shellfish Gathering and Shell Midden Archaeology". Advances in Archaeological Method and Theory, Vol. I0: $93-210$.

Zohar, I. y M. Belmaker. 2005. "Size Does Matter: Methodological Comments on Sieve Size and Species Richness in Fishbone Assemblages". Journal of Archaeological Science 32: 635 641.

Yesner, D.R. 1980. "Maritime hunter-gatherers: ecology and prehistory". Current Anthropology 21: 727 - 750.

Zúñiga, O. 2002. Moluscos: Guía de Biodiversidad. Vol. I. CREA, Universidad de Antofagasta, Antofagasta. 